\title{
Einführung - Entwicklungspolitik - über die Ankündigungspolitik hinaus
}

\section{Catherine Schümperli Younossian}

\section{(2) OpenEdition}

\section{Journals}

Electronic version

URL: http://journals.openedition.org/sjep/134

DOI: $10.4000 /$ sjep. 134

ISSN: 1663-9677

Publisher

Institut de hautes études internationales et du développement

Printed version

Date of publication: 1 avril 2006

Number of pages: XIII-XVIII

ISBN: 2-88247-062-2

ISSN: $1660-5926$

\section{Electronic reference}

Catherine Schümperli Younossian, « Einführung - Entwicklungspolitik - über die Ankündigungspolitik hinaus », Schweizerisches Jahrbuch für Entwicklungspolitik [Online], 25-1 | 2006, Online erschienen am: 15 April 2010, abgerufen am 25 September 2020. URL : http://journals.openedition.org/sjep/134 DOI : https://doi.org/10.4000/sjep.134 


\section{Einführung}

\section{Entwicklungspolitik - über die Ankündigungspolitik hinaus}

I M JAHR 2005 sind der Öffentlichkeit fünf wichtige Tatsachen bewusst geworden, die auf eine neue und tief greifende Solidarität gegenüber den Ländern des Südens hoffen lassen: Die grosse Mobilisierung der internationalen Gemeinschaft nach dem Tsunami, der im Dezember 2004 die Küsten des Indischen Ozeans verwüstete, die Aufhebung der multilateralen Schulden 18 schwer verschuldeter Länder, die von der G-8 an ihrem Treffen im Juni 2005 beschlossen wurde, das am Millennium+5-Gipfel erneut bekräftigte Engagement der Staatschefs, effizient gegen die Armut vorzugehen, die Weiterführung einer Verhandlungsrunde über den Welthandel zugunsten der Entwicklung, und schliesslich, in der Schweizer Politik, die Bekanntgabe des Bundesrates, dass das Ziel, 0,4 Prozent des Schweizer Bruttonationaleinkommens für die öffentliche Entwicklungshilfe einzusetzen, erreicht wurde.

Wie gross ist die Tragweite dieser Ereignisse wirklich? Sind es politische Ankündigungen ohne Folgen? Oder anders gefragt: Auf welche Schwierigkeiten stösst man bei der Umsetzung solcher Beschlüsse? Wie weit können diese Ankündigungen Auswirkungen auf das Verständnis und die Wahrnehmung der heutigen Probleme in der breiten Öffentlichkeit haben?

Als Antwort auf diese Fragen legt das Schweizerische Jahrbuch für Entwicklungspolitik. Fakten und Statistiken Elemente der Information und der Analyse zu allen Beziehungen zwischen der Schweiz und den Ländern des Südens und des Ostens vor. Durch seinen Ansatz, und indem es die so ermittelten Ereignisse in die richtige Perspektive rückt, versucht es, über die Grenzen einer allzu kurzfristigen Information hinauszugehen. Dazu wird näher auf die fünf genannten Beispiele eingegangen, um die Herausforderungen, vor welche uns die Fragen der Entwicklungspolitik stellen, aber auch die Komplexität ihrer Umsetzung besser verständlich zu machen.

\section{Tsunami - Ausdruck einer aussergewöhnlichen internationalen Solidarität?}

Kein Zweifel, die Solidaritätswelle war aussergewöhnlich! Die zur Verarbeitung der Folgen dieser humanitären Katastrophe versprochenen Gelder brachen alle Rekorde. Ein Jahr nach der Jahrhundertflut, die im Dezember 2004 in gut zehn Anrainerländern des Indischen Ozeans Hunderttausende von Todesopfern gefordert hat, ist es nun aber Zeit, die Gründe zu analysieren, welche zu dieser privaten wie öffentlichen internationalen Grosszügigkeit geführt haben, aber auch die Schwierigkeiten vor Ort, als es darum ging, die Hilfe effizient zu koordinieren.

Im Februar 2005 überstieg die von den Geberländern versprochene Hilfe 11 Milliarden Dollar, und die Solidarität von privater Seite legte Zeugnis ab von der Bereitwilligkeit der Öffentlichkeit, auf Spendenaufrufe für humanitäre Einsätze zu reagieren. In der Schweiz sammelte die Glückskette über 226 
Millionen Schweizer Franken. Der ausserordentliche Umfang der Katastrophe und die darauf folgende breite Berichterstattung in den Medien erklären diese Solidaritätswelle zumindest teilweise.

Ist das Geld einmal eingegangen, stellt sich die heikle Frage, wie es in den von der Katastrophe betroffenen Ländern verwendet werden soll. Die hohe Zahl der Akteure (Regierungen, multilaterale Agenturen und NRO) führte zu reellen Problemen bei der Koordinierung und der „Absorptionskapazität“ der Hilfe, wozu noch der Umgang mit ungeeigneten Spenden kam.

Doch darf diese grosse Solidaritätswelle andere humanitäre Krisen nicht vergessen lassen, die bei den Medien auf weniger Echo stossen, was das Zusammentragen der nötigen Finanzen erschwert. Die humanitären Tragödien nach dem Erdbeben in der Region Kaschmir im Oktober 2005, die Verwüstungen des Hurrikans Stan in Mittelamerika fast zur gleichen Zeit sowie die Krise in Darfur illustrieren diese dramatische Realität. Auch der Aufruf zahlreicher Fachleute aus Entwicklungskreisen muss erwähnt werden, welche bei dieser Rekordspendensammlung daran erinnerten, dass es auch „stille Tsunamis“ gibt, die Woche für Woche mehr Todesopfer fordern als die Flutwelle vom Dezember 2004 Todesopfer, welche die Folge so heimtückischer Übel wie Armut, Hunger und ansteckende Krankheiten sind. (Kapitel 4 - Humanitäre Hilfe.)

\section{Entschuldung für die Länder des Südens -} Ein Resultat von historischer Tragweite?

Im Juni 2005 kündigten die Finanzminister der reichen Länder am Gipfel der G-8 an, dass sie 18 armen Ländern die multilateralen Schulden in Höhe von 40 Milliarden Dollar erlassen wollten. Es geht dabei um die Mehrfachverschuldung dieser Länder beim Internationalen Währungsfonds (IWF), der Weltbank (WB) und der Afrikanischen Entwicklungsbank (AfDB). Hierzu ist zu vermerken, dass die Länder, die von dieser Massnahme profitieren, tatsächlich niemals in der Lage wären, ihre Schulden zurückzuzahlen. Deshalb mussten die Gläubiger ihre Strategie ändern. Einer der problematischen Aspekte dieser Verpflichtung betrifft die Gläubigerländer, die sie umsetzen müssen, die aber weder am Beschluss noch an der Ankündigung (!) beteiligt waren. Die Initiative wurde unter dem Namen Multilateral Debt Relief Initiative auf multilateraler Ebene lanciert, was die anderen Länder der internationalen Gemeinschaft, auch die Schweiz, praktisch dazu zwingt, sie mitzutragen.

Ausserdem ist nirgends festgehalten, dass bei dieser mit grossem Medieneinsatz angekündigten Initiative Einzelheiten über die Modalitäten der Finanzierung fehlen. Wird sie über zusätzliche Gelder finanziert oder sollen als Ersatz andere Budgetposten der öffentlichen Entwicklungshilfe belastet werden (Kürzung der bilateralen Hilfe oder der Beiträge an multilaterale Organisationen)? Ersteres scheint eher unwahrscheinlich, auch für jene Länder, die hinter der Initiative stehen. Deshalb besteht eine reale Gefahr, dass der zweite Fall eintritt, wodurch das Erreichen der Millenniums-Entwicklungsziele (Millennium Development Goals, MDG) noch stärker gefährdet wird.

Zwar unterstützt die Schweiz das Prinzip dieser Initiative, eine konkrete Teilnahme zieht sie aber erst in Betracht, wenn die genauen Modalitäten und die 
finanziellen Auswirkungen bekannt sind. Bei den Finanzinstitutionen sieht die Situation kaum besser aus: Der IWF hat beschlossen, für die Finanzierung der G-8-Initiative rund 4,8 Milliarden Dollar einzusetzen, die Weltbank und die Afrikanische Entwicklungsbank haben bis Ende 2005 noch nichts Näheres über die Modalitäten ihres Engagements bekannt gegeben. (Kapitel 7 - Internationale Finanzbeziehungen.)

Millenniumsgipfel - Können nach der feierlichen Verpflichtung der Staatschefs die Millenniums-Entwicklungsziele erreicht werden?

Im September 2005, fünf Jahre nach der Unterzeichnung der Millenniumserklärung für die Entwicklung, die acht quantifizierbare Ziele im Kampf gegen die Armut anstrebt, bekräftigten die Staatschefs von 191 Ländern erneut ihre Verpflichtung zur Armutsbekämpfung. In den letzten fünf Jahren versuchten internationale Organisationen, Agenturen für bilaterale Zusammenarbeit und NRO ohne grossen Erfolg, sich auf die gesteckten Ziele einzustellen.

Anlässlich des Gipfels von 2005 musste eine traurige Bilanz gezogen werden: Nein, die Millenniums-Entwicklungsziele können in den meisten Entwicklungsländern nicht erreicht werden. Unter den 47 afrikanischen Ländern dürften die meisten den gesetzten Zeitplan nicht einhalten können, sie werden also die extreme Armut bis 2015 nicht wesentlich abbauen und auch keine nennenswerten Fortschritte in den Bereichen Gesundheit, Erziehung und Umweltschutz erzielen.

Abgesehen von dieser zumindest gemischten Bilanz stellen sich einige grundlegende Fragen zum Begriff der Millenniums-Entwicklungsziele selber. Zwar ist die Einführung quantifizierbarer Ziele, dank denen die Fortschritte gemessen werden können, für die Bekämpfung der Armut begrüssenswert. Allerdings lässt die MDG-Initiative verschiedene Interpretationen $\mathrm{zu}$, welche unbedingt geklärt werden müssen. Sollen es Ziele sein, die um jeden Preis erreicht werden müssen, weil das Ganze sonst in einem Misserfolg endet? In diesem Fall ist zu befürchten, dass eine nicht immer realistische Definition der Ziele ein pessimistisches Klima rund um die Entwicklungshilfe schafft. Oder müssen die Ziele vielmehr ein Instrument sein, das mithilft, die Diskussion über den Zugang zu den sozialen Grunddiensten zu eröffnen, die Beträge der öffentlichen Entwicklungshilfe zu erhöhen und die Öffentlichkeit für das Ungleichgewicht zwischen Nord und Süd zu sensibilisieren? Die Interpretation der Millenniums-Entwicklungsziele muss unbedingt klargestellt werden, da es sonst zu einer grossen Desillusionierung in der Öffentlichkeit kommt. (Kapitel 2 - Zusammenarbeit mit den Entwicklungsländern.)

\section{WTO-Verhandlungen - Dient die Doha-Runde wirklich der Entwicklung?}

„,Vergessen wir nicht, dass der Handel nur ein Werkzeug zur Verbesserung der Lebensbedingungen ist: Im Mittelpunkt unserer Überlegungen sollten letztendlich immer die Folgen stehen, die unsere Regeln für die Menschen haben." Diesen Appell lancierte der Generaldirektor der Welthandelsorganisation (WTO) am 30. Januar 2006 in einer Rede über die „Humanisierung der Globalisierung“, 
die er in Santiago de Chile hielt. Es ist aber zu vermerken, dass die in der DohaRunde eingeleiteten Verhandlungen nur mit Mühe abgeschlossen werden können, vielleicht wegen des Paradigmenwechsels, wonach die Doha-Runde zu einer „Entwicklungsrunde“ werden soll. Und das ist genau der wunde Punkt: Der Sinn, den man dem Wort „Entwicklung“ gibt, ändert sich je nach den betroffenen Akteuren.

Zwei Beispiele, von denen 2005 in den Medien viel die Rede war, zeugen von dieser Interpretationsschwierigkeit. Sie dürfen aber andere, ebenso heikle Bereiche nicht vergessen lassen. Das erste Beispiel betrifft den erleichterten Zugang der Entwicklungsländer zu den Agrarmärkten der Industriestaaten. In diesem Sektor sind die strukturellen Ungleichheiten zwischen den Industriestaaten und den Entwicklungsländern frappant. Für die Länder des Südens, welche Landwirtschaftsprodukte ausführen und in der G-20 zusammengefasst sind, bedeutet eine „Entwicklungsrunde“, die ihren Namen verdient, dass die reichen Länder eine Öffnung im Agrarbereich zulassen, ohne dafür massive Konzessionen in anderen Bereichen zu fordern. Dies jedoch lehnen die Industriestaaten $a b$, sie sind $\mathrm{zu}$ einer eventuellen Öffnung in der Landwirtschaft nur bereit, wenn die Entwicklungsländer im Gegenzug ihren Industriegütern und Dienstleistungen einen besseren Zugang zu ihren Märkten gewähren.

Das zweite Beispiel betrifft den Zugang der Entwicklungsländer zu den Medikamenten. Wir erinnern daran, dass in den Entwicklungsländern 95 Prozent der Aidskranken zu einem vorschnellen Tod verurteilt sind, weil sie keinen Zugang $\mathrm{zu}$ antiretroviralen Behandlungen haben. Das Problem stellt sich auch Millionen von Menschen, welche an Krankheiten leiden, die in den Industriestaaten heilbar sind. So müssen, auch aus Gründen der Volksgesundheit, unbedingt die Rechte der Entwicklungsländer bei der Nutzung von Zwangslizenzen gestärkt werden. Ausserdem müssen diese Länder die Möglichkeit erhalten, Generika patentgeschützter Medikamente einführen zu können. Dazu wurde zwar im August 2003 ein Beschluss gefasst, doch ist dieser nicht sehr befriedigend, weil die WTO - namentlich unter dem Druck der USA und der Schweiz - das Zugangsrecht zu Generika Einschränkungen unterstellt hat, die von den armen Ländern, insbesondere jenen in Afrika südlich der Sahara, heftig angefochten werden.

Und schliesslich ein letztes Element dieser Schwierigkeit, dem Begriff „Entwicklung" einen Sinn zu geben: An der letzten WTO-Ministerkonferenz, die im Dezember 2005 in Hongkong stattfand, waren praktisch alle Delegationen, die an den Verhandlungen teilnahmen, aus Unterhändlern zusammengesetzt, die aus dem Handel kommen. Delegierte, welche in Entwicklungsfragen bewandert sind, waren eine Seltenheit. Immerhin gehörten der gut fünfzehnköpfigen Schweizer Delegation erstmals zwei Delegierte der Direktion für Entwicklung und Zusammenarbeit (DEZA) an. Sie verstärkten damit diese Dimension, die bis dahin von zwei Delegierten der Sektion Entwicklungszusammenarbeit des Staatssekretariats für Wirtschaft (seco) vertreten war. Allerdings handelt es sich bei den Verhandlungszielen weiterhin um Handelsbereiche, und die Berücksichtigung der Entwicklungsdimension ist im Moment nicht gegeben, oder - noch schlimmer - sie bleibt höchstens symbolisch. (Kapitel 6 - Welthandel.) 

wirklich erreicht?

International ist der wichtigste Indikator des Engagements der Industriestaaten zugunsten der Entwicklungszusammenarbeit der Prozentsatz des Bruttonationaleinkommens (BNE), der für die öffentliche Entwicklungshilfe eingesetzt wird. Zwar bleibt das Ziel von 0,7 Prozent des BNE der Massstab, auf den sich die internationale Gemeinschaft bezieht, es wird aber erst von fünf Ländern erreicht.

Die Schweiz hatte 1992 an der Konferenz über Umwelt und Entwicklung in Rio angekündigt, sie wolle in spätestens zehn Jahren 0,4 Prozent ihres BNE für das Budget der Entwicklungszusammenarbeit einsetzen. 2005 kann sie ihr Versprechen endlich halten, doch musste eine neue Berechnungsmethode für die öffentliche Entwicklungshilfe angewandt werden, um das Ziel zu erreichen. Der Bundesrat hat in der Tat im Mai 2005 den Beschluss gefasst, die Ausgaben, die für die Asylbewerber aus Entwicklungsländern in ihrem ersten Jahr in der Schweiz getätigt werden, in die Berechnung der öffentlichen Entwicklungshilfe einzubeziehen. Dank diesem statistischen „Trick“ kann die Schweiz die an der Konferenz von Rio eingegangene und in offiziellen Berichten oft bekräftigte moralische Verpflichtung erfüllen.

So ist die öffentliche Entwicklungshilfe der Schweiz nach verschiedenen Buchhaltungsrevisionen nominell von 1,75 Milliarden Schweizer Franken im Jahr 2003 auf 1,92 Milliarden im Jahr 2004, oder in Prozenten ausgedrückt, von 0,39 auf 0,41 Prozent des Bruttonationaleinkommens gestiegen. Damit steht die Schweiz unter den 22 OECD-Ländern an achter Stelle. Zwar dürfte die Verbuchung der Hilfe an die Asylbewerber in den Ausgaben der öffentlichen Entwicklungshilfe weitergehen (neun Länder schliessen die Kosten für den Unterhalt der Asylbewerber ganz oder teilweise ein), doch haben diese Massnahmen natürlich keinen Einfluss auf das effektive Budget der Schweizer Entwicklungszusammenarbeit. (Kapitel 2 -Zusammenarbeit mit den Entwicklungsländern.)

* * *

Ein herzlicher Dank des Redaktionskomitees geht an die Autorinnen und Autoren der im vorliegenden Band Schweizerisches Jahrbuch für Entwicklungszusammenarbeit veröffentlichten Texte sowie an die zahlreichen Spezialisten in den Bundesbehörden, Entwicklungs-NRO und Akademikerkreisen, welche durch ihre kritische Überprüfung und ihre wertvollen Ratschläge unserer Publikation zu ihrer grossen Seriosität verholfen haben. Unser Dank geht auch an all jene, die zur Gestaltung, Ausführung und Übersetzung des Werks beigetragen haben. Auf ihrer Kompetenz und ihrem Engagement beruht die Zuverlässigkeit dieses Nachschlagewerks.

Zum Schluss nimmt das Redaktionskomitee mit Bedauern Abschied von Professor Jacques Forster, der mit Erreichen des Pensionsalters als Redaktionsleiter zurücktritt, und spricht ihm seine grosse Anerkennung aus. Jacques Forster war Gründer der vorliegenden Sammlung und Leiter des Werks Jahrbuch Schweiz Dritte Welt, das 2003 in Schweizerisches Jahrbuch für Entwicklungspolitik umbenannt wurde. Er leitete die Veröffentlichung während nahezu fünfundzwanzig Jah- 
ren. Streng und grosszügig zugleich gelang es ihm, seine Begeisterung auf seine Mitarbeiterinnen und Mitarbeiter zu übertragen, er liess sie Anteil haben an seinem lebhaften Interesse und seinem immensen Wissen zu den Nord-Süd-Beziehungen im Allgemeinen und den Beziehungen „Schweiz - Dritte Welt“ im Besonderen. Das ganze Team des Jahrbuchs dankt ihm von Herzen für die Jahre seines Engagements und wünscht ihm für den neuen Lebensabschnitt alles Gute.

Catherine Schümperli Younossian Forschungsbeauftragte am IUED, Redaktionsleiterin 